\title{
The oral surgeon in the front-line of the diagnosis of Ewing sarcoma: a case report
}

\author{
Myriam Saleh", Ludwig Loison-Robert, Véronique Guillerminet, Patrick Larras, \\ Alexandre Berquet, Victorin Ahossi
}

Odontology Department - University Hospital Center, Dijon, France

(Received: 21 February 2018, accepted: 26 April 2018)

Keywords:

Ewing sarcoma / cellulitis / mandible

\begin{abstract}
Introduction: Ewing's sarcoma is a primary malignant bone tumor that affects children and young adults. In rare cases, it affects the bones of the face. The diagnosis is difficult because signs are non-specific. Positive diagnosis is based on a cytogenetic examination showing a specific translocation of the EWS gene. Treatment involves a sequence including chemotherapy, oncological surgery and radiotherapy. Observation: The case presented is that of a 4-year old girl initially sent for suspicion of dental cellulitis. On clinical examination, she had recent mandibular dental mobility and a gingival mucosa swelling. Signs of bone lysis interesting the mandibular symphysis was observed on the orthopantomogram. She had no pain or symptoms indicating neoplasia. This atypical situation led to a bone biopsy who revealed the diagnostic of mandibular Ewing's sarcoma. A suitable medical care in a specialized unit was able to be started without delay. Discussion: The literature reports other cases also wrongly diagnosed as an infectious pathology of dental origin. Conclusion: This case shows that the diagnosis of mandibular Ewing's sarcoma must be evoked in front of any atypical mandibular lesion of the child, regardless of his age or sex. Physicians and dental surgeons specialized in oral surgery are then in the foreground to help establish early diagnosis and ensure rapid management by limiting the functional and aesthetic sequelae related to localization.
\end{abstract}

\section{Introduction}

Ewing's sarcoma is the second most common primary bone tumor in children and young adults [1]. It occurs preferentially in males between 5 and 20 years of age and only affects the facial bones in $2-3 \%$ cases and may have a misleading presentation $[2,3]$. The prognosis is primarily related to the presence of metastases and it is important to establish an early diagnosis despite some unspecific signs [4-7]. The case reported is that of a 4-year-old girl who was referred to the dentistry emergency departments of the university hospital center by her oral surgeon with an initial diagnosis of dental cellulitis that was not responding favorably after 1 week of antibiotic treatment.

\section{Observation}

During clinical history taking, the mother reported a progressive displacement of the anterior mandibular teeth over a 4-month period. In the few days before presentation, she noted the appearance of a slight swelling in the left submandibular area, accompanied by the loss of the tooth 71 .

\footnotetext{
*Correspondence: salehmyriam99@hotmail.fr
}

Upon admission, she did not have any systemic symptoms. An extraoral examination revealed a painless left mandibular swelling without any changes in sensitivity in the lower alveolar nerves.

Intraorally, there was mobility and distal displacement of teeth $81,82,72,73$. There was also budding gingival mucosa at the site of the tooth 71 and teeth 74 to 83 did not respond to the cold test (Fig. 1). The noninflammatory appearance of the swelling and the absence of pain allowed us to rule out an acute infection. Coronal reconstruction of the cone-beam computed tomography revealed an ill-defined hypodense osteolytic lesion extending from the right para-symphyseal region to the left horizontal branch. Teeth $81,82,72$, and 73 were distally displaced.

Apical resorption of the teeth in contact with this lytic lesion was observed. The germs of the permanent teeth were displaced with basilar cortical erosion in the median area (Fig. 2). The axial section also showed vestibular cortical lysis, a spicular periosteal reaction, vestibular displacement of the permanent tooth germs, and thickening of the adjacent soft tissues (Fig. 3). All images show nonspecific lesions of aggressive appearance. A biopsy of the lesion was thus performed under general anesthesia to facilitate diagnosis. 


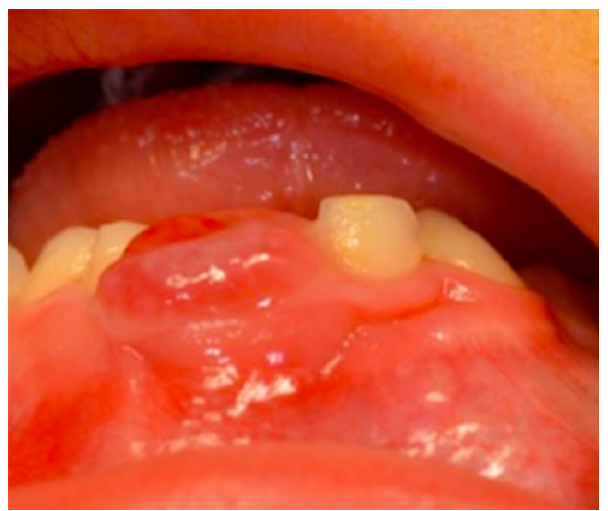

Fig. 1. Endo-oral examination: budding gingival mucosa at the site of the left deciduous central incisor.

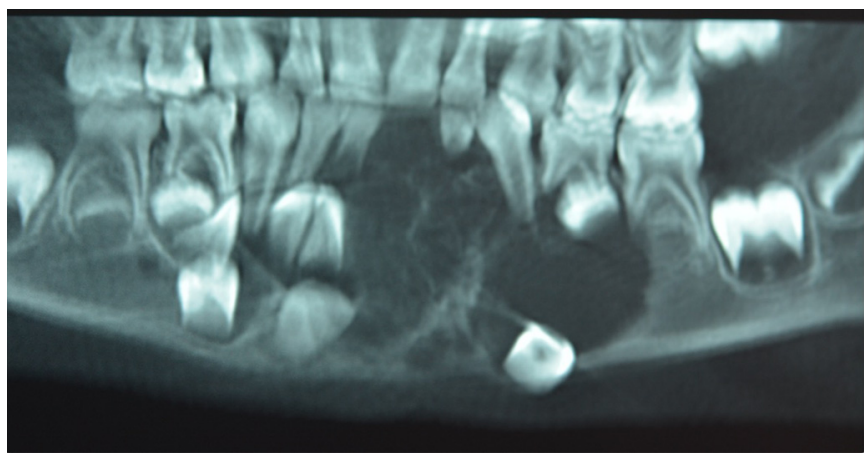

Fig. 2. Cone-beam computed tomography, mandibular sector: multiplanar reconstruction, coronal view: hypodense osteolytic lesion from the right parasymphyseal region to the left horizontal limb, suggestive of an aggressive tumor (poorly limited, with resorption, and displacement of the teeth in contact).

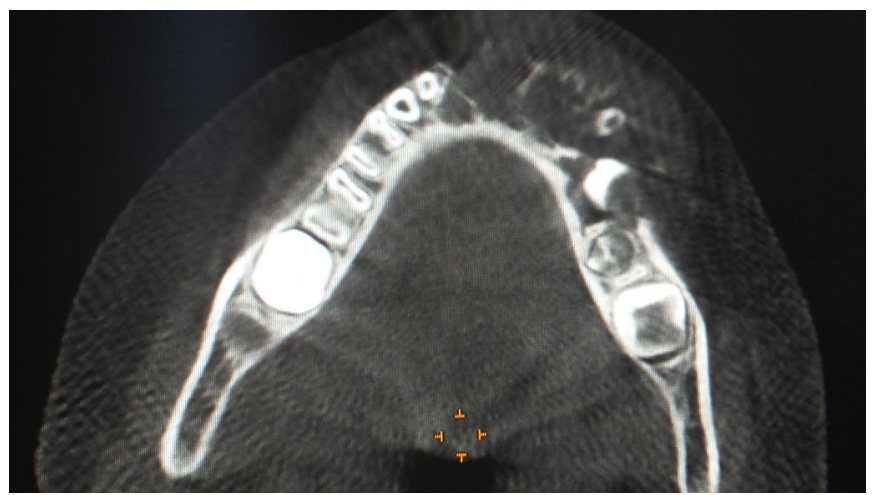

Fig. 3. Cone-beam computed tomography. Axial view: vestibular cortical lysis, spike periosteal reaction, vestibular displacement of tooth germs and thickening of adjacent soft tissues.

The bone showed lysis and infiltration. A histopathological examination showed small round monomorphic blue cells with rounded and oval nuclei containing fine chromatin (Fig. 4). Immunohistochemistry revealed specific labelling of anti-CD99 and anti-synaptophysin. The cytogenetic study via the FISH

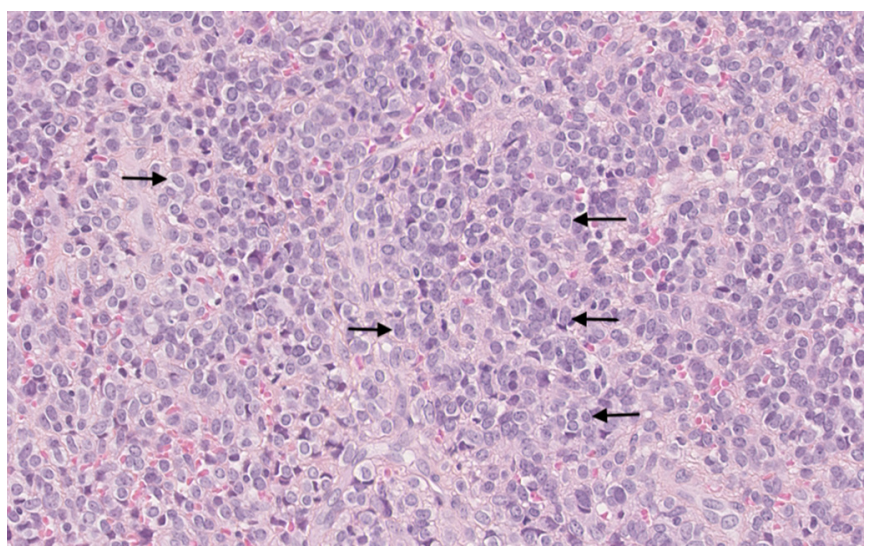

Fig. 4. Anatomopathological examination HES $\times 40$. Monomorphic proliferation of "blue" tumor cells with oval or rounded nuclei, medium size, surrounded by a scanty eosinophilic cytoplasm, sometimes clarified, poorly defined (Arrows).

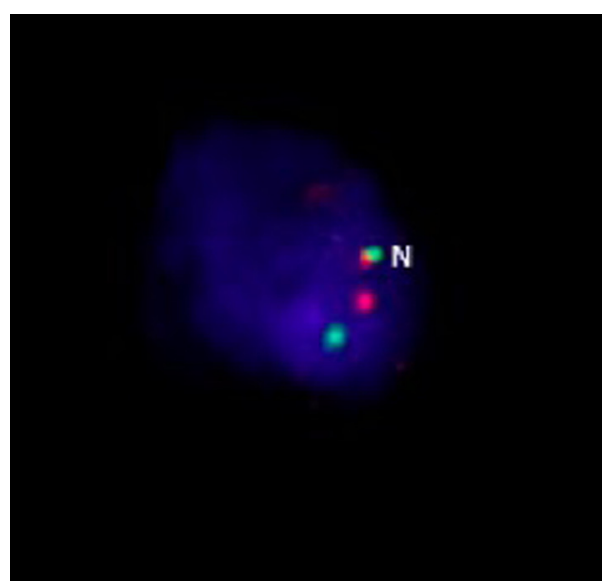

Fig. 5. Cytogenetic finding: FISH (indirect immunofluorescence in situ hybridization) method on paraffin section. A normal two-color signal $(\mathrm{N})$, a red signal and a separate green signal highlighting one of the specific translocations of the EWS gene.

technique (in situ hybridization by indirect immunofluorescence) revealed one of the specific translocations of the EWS gene, definitively confirming the diagnosis of Ewing's sarcoma (Fig. 5). Locoregional extension assessment including magnetic resonance imaging, cervical CT scan, bone scan, positron emission tomography scan and osteomedullary biopsy for metastasis was negative.

At the biological level, the initial assessment showed no disturbance except anemia at $10.8 \mathrm{~g} / \mathrm{dl}$. As soon as the results were available, the girl was referred to a pediatric oncology department for multidisciplinary care following a diagnostic consultation.

Its management was carried out in accordance with the Euro Ewing 2012 protocol including neoadjuvant chemotherapy, oncological surgery after tumor volume reduction, radiotherapy according to the resection margins, and adjuvant chemotherapy. 


\section{Discussion}

Ewing's sarcoma is an aggressive, primitive, malignant bone tumor, first described by James Ewing in 1921 [1]. It affects children and young adults preferentially between 5 and 20 years of age, with a male predominance of (2:1) [1] and preferably persons of Caucasian origin $[6,8]$. It is the second most common childhood tumor after osteosarcoma, accounting for $1 \%$ of all childhood tumors. It is highly chemosensitive, and its management is multimodal, combining neoadjuvant chemotherapy, surgery, margin-based radiotherapy and adjuvant chemotherapy [9]. The presence of an initial spread is the main prognostic factor.

Overall survival is $60 \%$ at 5 years if there is no remote localization, and it decreases by half in the presence of initial metastases $[10,11]$. The most frequent localization is the diaphysis of the long bones, the pelvis, and the ribs. The face is rarely involved, and the mandible represents only $0.7 \%$ of all the locations observed with a clinical presentation that can be misleading $[1,5]$. In its most common classic presentation, Ewing's sarcoma is manifested as a clinical tumor syndrome with associated pain, fever, systemic impairment, and pathological fractures [9].

At the mandibular level, the symptoms are inconsistent and nonspecific like a more or less painful swelling that can be mistaken for a dental infection and thus delay the tumor diagnosis. In the literature, cases of mandibular localization are rare and most often affect the posterior part such as the ramus or ascending branch [12-14]. According to a literature review published in 2017, 21\% mandibular Ewing sarcomas are initially misdiagnosed as infectious diseases of dental origin because of their nonspecific manifestations. Indurated swelling has been reported in $70 \%$ of cases and $<30 \%$ of the persons affected experience pain [13]. Fever and lymphadenopathy, which are frequent in the usual locations, are found in $<10 \%$ of cases. Paresthesia may also occur near the inferior alveolar nerve as well as trismus. Dental symptoms are common and are accompanied by movement and mobility. The mucus membrane is often healthy or erythematous and is rarely ulcerated [13].

\section{Conclusion}

Ewing's sarcoma is a highly aggressive, poorly differentiated tumor that very rarely affects the mandible. Due to the high metastatic potential that conditions the prognosis, it is important not to delay the diagnosis. Often, the only symptoms are swelling and osteolysis as seen on the orthopantomogram. The main problem with Ewing mandibular sarcomas is local treatment. Surgery usually consists of a total or subtotal mandibulectomy that must be performed with healthy margins to allow immediate reconstruction by flap or prosthesis. Radiotherapy causes growth disorders, increases the risk of radiation-induced tumors, and decreases the chances of successful mandibular reconstruction techniques. Early treatment increases the chances of having a surgical resection with healthy margins, which avoids the need for radiotherapy and its functional and esthetic consequences. Thus, any suspicious imaging finding of osteolysis in children and young adults should be further investigated with more detailed imaging without delay to dispel any doubt and a biopsy to confirm diagnosis and allow prompt referral to a specialty center.

Conflicts of interest: The authors declare that they have no conflicts of interest in relation to this article.

\section{References}

1. Lopes SLPC, Almeida SM de, Costa ALF, Zanardi VA, Cendes F. Imaging findings of Ewing's sarcoma in the mandible. J Oral Sci 2007;49:167-171.

2. Keshani F, Jahanshahi G, Attar BM, Kalantari M, Razavi SM, Hashemzade Z, Tavakoli P. Ewing's sarcoma in mandibular similar to dental abscess. Adv Biomed Res 2014;3:62.

3. Davido N, Rigolet A, Kerner S, Gruffaz F, Boucher Y. Case of Ewing's sarcoma misdiagnosed as a periapical lesion of maxillary incisor. J Endod 2011;37:259-264.

4. Ko E, Brouns ER, Korones DN, Pochal WF, Philipone EM, Zegarelli DJ, Yoon AJ. Primary Ewing sarcoma of the anterior mandible localized to the midline. Oral Surg Oral Med Oral Pathol Oral Radiol 2013;115:e46-e50.

5. Rao BS, Rai G, Hassan S, Nadaf A. Ewing's sarcoma of the mandible. Natl J Maxillofac Surg 2011;2:184-188.

6. Gosau M, Baumhoer D, Ihrler S, Kleinheinz J, Driemel 0. Ewing sarcoma of the mandible mimicking an odontogenic abscess $-\mathrm{a}$ case report. Head Face Med 2008;4:24.

7. Akbayram S, Basaranoglu M, Kaya A, Açıkgöz M, Üstyol L, Taskın G, Dogan M. Ewing's sarcoma localized in the mandible: a case report. West Indian Med J 2015;64:444-446.

8. Gupta S, Gupta OP, Mehrotra S, Mehrotra D. Ewing sarcoma of the maxilla: a rare presentation. Quintessence Int 2009;40: 135-140.

9. Hernandez M, Droz D, Mansuy L, Simon E, Chastagner P. Ewing sarcoma located in the mandible: a case report. Arch Pediatr 2015;22:626-629.

10. Berk R, Heller A, Heller D, Schwartz S, Klein EA. Ewing's sarcoma of the mandible: a case report. Oral Surg Oral Med Oral Pathol Oral Radiol Endod 1995;79:159-162.

11. Krishna KB, Thomas V, Kattoor J, Kusumakumari P. A radiological review of Ewing's sarcoma of mandible: a case report with one year follow-up. Int J Clin Pediatr Dent 2013;6:109-114.

12. Da Fonseca MA, Abrams RB. Ewing's sarcoma of the mandible in a young patient: case report. Pediatr Dent 1992;14: 402-404.

13. Margaix-Muñoz M, Bagán J, Poveda-Roda R. Ewing sarcoma of the oral cavity, a review. J Clin Exp Dent 2017;9:e294-e301.

14. Van den Bergh JP, Van der Waal I. Ewing's sarcoma of the mandible: report of a case. J Oral Maxillofac Surg 1988;46: 798-800. 